\title{
Suberoylanilide hydroxamic acid upregulates histone acetylation and activates endoplasmic reticulum stress to induce apoptosis in HepG2 liver cancer cells
}

\author{
LEI YU ${ }^{1,2^{*}}$, RUJIA XIE $^{1,3^{*}}$, TIAN TIAN $^{1,3}$, LU ZHENG $^{1,3}$, LEI TANG $^{1,3}$, SHUANG CAI $^{1,3}$, \\ ZIHUA MA ${ }^{1,3}$, TING YANG ${ }^{1,3}$, BING HAN ${ }^{1,3}$ and QIN YANG ${ }^{1,3}$ \\ ${ }^{1}$ Department of Pathophysiology, College of Basic Medical Sciences, Guizhou Medical University, \\ Guiyang, Guizhou 550025; ${ }^{2}$ Department of Pathology, Hospital of Maternal and Childhood Health, \\ Guiyang, Guizhou 550000; ${ }^{3}$ Guizhou Provincial Key Laboratory of Pathogenesis and Drug Research on \\ Common Chronic Diseases, Guizhou Medical University, Guiyang, Guizhou 550025, P.R. China
}

Received December 5, 2018; Accepted June 26, 2019

DOI: $10.3892 / \mathrm{ol} .2019 .10705$

\begin{abstract}
Suberoylanilide hydroxamic acid (SAHA) is a histone deacetylase inhibitor that has demonstrated clinical activity against various solid tumors. The aim of the present study was to explore the effects of SAHA on the apoptosis of HepG2 liver cancer cells, as well as the potential mechanisms involved in histone acetylation and endoplasmic reticulum (ER) stress. HepG2 cells were treated with various doses of SAHA $(0,1,6$ and $12 \mu \mathrm{M})$, and apoptosis was measured by flow cytometry. The levels of ER stress-associated molecules, including $78 \mathrm{kDa}$ glucose-regulated protein (GRP78), PRKR-like endoplasmic reticulum kinase (PERK), phosphorylated (p)-PERK, activating transcription factor 4 (ATF4) and $\mathrm{C} / \mathrm{EBP}$-homologous protein (CHOP), were quantitated by western blot analysis and reverse transcription-quantitative PCR assay. The expression levels of acetylated histone H4 (acH4, acH4 lysine (K)5 and acH4K12) were detected by western blot analysis. The effects of SAHA on the acetylation of $\mathrm{H} 4$ in the promoter regions of GRP78, ATF4 and CHOP were evaluated by chromatin immunoprecipitation assays. Following treatment with higher doses of SAHA (6 and $12 \mu \mathrm{M}$ ) for $48 \mathrm{~h}$, the proliferation of HepG2 cells was significantly suppressed. SAHA induced dose-dependent apoptosis and increased both protein and mRNA expression
\end{abstract}

Correspondence to: Dr Bing Han or Dr Qin Yang, Department of Pathophysiology, College of Basic Medical Sciences, Guizhou Medical University, 3 Dongqing Road, Guiyang, Guizhou 550025, P.R. China

E-mail: 47569390@qq.com

E-mail: qinyang@gmc.edu.cn

*Contributed equally

Key words: suberoylanilide hydroxamic acid, histone deacetylase inhibitor, liver cancer, HepG2, apoptosis, endoplasmic reticulum stress, histone $\mathrm{H} 4$ levels of GRP78, ATF4 and CHOP in HepG2 cells. The protein expression of PERK was markedly decreased by treatment with SAHA, whereas the p-PERK expression level was notably increased, which resulted in increased p-PERK/PERK ratio. Furthermore, the acetylation levels of $\mathrm{H} 4$ in the promoter regions of GRP78, ATF4 and CHOP were significantly increased in HepG2 cells exposed to $6 \mu \mathrm{M}$ SAHA for $36 \mathrm{~h}$. Thus, SAHA induces apoptosis in HepG2 cells by activating the ER stress-mediated apoptotic signaling pathway, at least partially by enhancing the acetylation of histone $\mathrm{H} 4$ on the promoter regions of ER-stress associated genes, including GRP78, ATF4 and CHOP.

\section{Introduction}

Liver cancer is among the most prevalent malignancies in the world (1); it has a high degree of malignancy and a poor prognosis. However, relatively limited treatment approaches are available in the clinic. Therefore, there is an urgent need to identify novel therapeutic targets and to develop effective strategies for liver cancer therapy. Emerging evidence has demonstrated a key role for epigenetics in tumorigenesis (2-4). The occurrence and development of liver cancer is also dependent on epigenetics-related disorders of various signal transduction pathways (5). Epigenetic regulation is defined as genomic modifications of heritable factors that do not involve alterations in the DNA sequence and affect the transcriptional activity of genes instead (6). Several mechanisms of epigenetic regulation, including histone modification, noncoding RNAs and DNA methylation, have been extensively studied (7). Among these mechanisms, histone modification is one of the important modes of epigenetic regulation in liver cancer (8).

Histones are well-conserved and highly alkaline proteins, which appear as an octameric core and help to package and order DNA into nucleosomes. The N-terminal tails of histones extend beyond the nucleosome and undergo a variety of post-translational modifications, such as acetylation, methylation, ubiquitination and phosphorylation (9). Acetylation of histones is mediated through histone acetylases (HATs) and 
histone deacetylases (HDACs) (10-12). Some studies have unveiled the importance of the balance of HATs and HDACs for the expression of several key genes, including p21, Fas, $\mathrm{Bcl}-2$ and $\mathrm{Bax}$ (13). Once this balance is broken, an imbalance of gene transcription occurs, which may lead to tumorigenesis or abnormal cell proliferation. Moreover, the high expression of HDAC family members and the downregulation of histone acetylation in liver cancer tissues have been confirmed in certain studies $(14,15)$. Therefore, the inhibition of HDAC activity by an HDAC inhibitor (HDACi) is expected to be an effective approach for the treatment of liver cancer.

Suberoylanilide hydroxamic acid (SAHA), the first HDACi approved for clinical use by the US Food and Drug Administration, has been widely acknowledged for its antitumor activity (16). It has been reported that SAHA decreases proliferation, induces differentiation and elicits apoptosis in many tumor cells during in vitro culturing (17-19). Additionally, SAHA has been demonstrated to be a potential inducer of endoplasmic reticulum (ER) stress that led to induction of apoptosis in lung cancer cells, mediated by the activation of the ER stress-mediated apoptotic signaling pathway (20). However, the effects of SAHA in liver cancer and the mechanism of SAHA in regulating ER stress are largely unknown.

In the present study, the effects of SAHA on apoptosis in the HepG2 liver cancer cells and the potential mechanisms involved in histone acetylation and ER stress were explored. Following treatment with various doses of SAHA, HepG2 cells were subjected to an apoptosis assay. Furthermore, the levels of the ER-stress related molecules $78 \mathrm{kDa}$ glucose-regulated protein (GRP78), activating transcription factor 4 (ATF4) and $\mathrm{C} /$ EBP-homologous protein (CHOP), as well as the acetylation levels of the histone proteins $\mathrm{H} 4(\mathrm{acH} 4), \mathrm{H} 4-1 y s i n e ~ 5$ (H4K5) and H4-lysine 12 (H4K12) were quantitated. The effects of SAHA on acetylation of $\mathrm{H} 4$ in the promoter regions of GRP78, ATF4 and CHOP were evaluated by chromatin immunoprecipitation (ChIP) experiments.

\section{Materials and methods}

Cell culture. The HepG2 liver cancer cell line (cat. no. KCB $200507 \mathrm{YJ}$ ) was purchased from the cell bank of the Type Culture Collection of the Chinese Academy of Sciences. The HepG2 cells were authenticated by short tandem repeat DNA profiling analysis and tested to be free of mycoplasma and other contaminants by the vendor. The cells were maintained in Dulbecco's modified Eagle's medium (Gibco; Thermo Fisher Scientific, Inc.) supplemented with $10 \%$ fetal bovine serum (ScienCell Research Laboratories, Inc.), $100 \mathrm{U} / \mathrm{ml}$ penicillin and $100 \mu \mathrm{g} / \mathrm{ml}$ streptomycin (Gibco; Thermo Fisher Scientific, Inc.) and grown in a humidified atmosphere with $5 \% \mathrm{CO}_{2}$ at $37^{\circ} \mathrm{C}$. The cells were treated with SAHA (Abcam) or mock-treated with DMSO (Sigma-Aldrich; Merck KGaA), as indicated.

Real-time cellular analysis. Cell proliferation was monitored using an xCELLigence Real-Time Cell Analysis instrument (xCELLigence DP System; ACEA Biosciences, Inc.), which can continuously monitor live cell proliferation, morphology and viability with a label-free assay. The experimental procedures used were as previously described $(21,22)$. Briefly, HepG2 cells were seeded at a density of $1 \times 10^{4}$ cells/well in a 16 -well $\mu$ l plate (E-plate 16), cultured in complete medium supplemented with various doses of SAHA $(0,0.1,1,6,12,25,50$ and $100 \mu \mathrm{M})$, and placed at $37^{\circ} \mathrm{C}$ in a humidified incubator containing $5 \% \mathrm{CO}_{2}$. The effects of the various doses of SAHA on the proliferation of HepG2 cells were continuously monitored for $90 \mathrm{~h}$. The cell index is a dimensionless parameter that is translated from the electrical impedance measured to denote cell proliferation.

Apoptosis assay. HepG2 cells $\left(1 \times 10^{6}\right)$ were seeded onto $10-\mathrm{cm}$ diameter dishes and treated with various doses of SAHA $(0,1,6$ and $12 \mu \mathrm{M})$ for $48 \mathrm{~h}$. The cells were harvested and subjected to an apoptosis assay using an annexin V/propidium iodide (PI) cell apoptosis detection kit (Nanjing KeyGen Biotech Co., Ltd.), according to the manufacturer's instructions. Briefly, the cells were washed with cold phosphate-buffered saline (PBS). After centrifugation at $110 \mathrm{~g}$ for $5 \mathrm{~min}$, the cell pellet was resuspended in $1 \mathrm{ml}$ PBS (final concentration $\sim 1-5 \times 10^{5}$ cells $/ \mathrm{ml}$ ). After washing with $1 \mathrm{X}$ annexin $\mathrm{V}$ binding solution, the cells were stained with $5 \mu 1$ of fluorescein isothiocyanate (FITC)-conjugated annexin V and $10 \mu \mathrm{l}$ PI staining solution at room temperature for $20 \mathrm{~min}$. After washing once with the $1 \mathrm{X}$ annexin $\mathrm{V}$ binding solution, the labeled cells $\left(1 \times 10^{4}\right.$ cells) were detected immediately by a flow cytometer (FACSCalibur ${ }^{\mathrm{TM}}$; BD Biosciences). The data were analyzed by BD CellQuest Pro software (version 1.0; BD Biosciences). The apoptotic rate was defined as the percentage of FITC-annexin $\mathrm{V}$ single positive cells (early apoptotic cells) among total cells.

Western blot analysis. HepG2 cells $\left(1 \times 10^{6}\right)$ were seeded onto $10-\mathrm{cm}$ diameter dishes and treated at various doses of SAHA $(0,1,6$ and $12 \mu \mathrm{M})$ for $48 \mathrm{~h}$. The cells were then lysed with $0.2 \mathrm{ml}$ of radioimmunoprecipitation assay buffer (Thermo Fisher Scientific, Inc.). Total protein samples $(60 \mu \mathrm{g})$ were separated by sodium dodecyl sulfate-polyacrylamide gel electrophoresis (10\% gels), transferred to a polyvinylidene difluoride membrane and blocked with $5 \%$ nonfat milk in Tris-buffered saline containing $0.1 \%$ Tween 20 (TBST) for $60 \mathrm{~min}$ at room temperature. The membrane was incubated overnight at $4^{\circ} \mathrm{C}$ with the following primary antibodies: acH4 (1:1,000), acH4K5 (1:5,000), acH4K12 (1:1,000), GRP78 (1:1,500), PERK (1:1,500), phosphorylated (p)-PERK $(1: 1,500)$, ATF4 $(1: 1,500)$ and CHOP $(1: 1,500)$. After washing with TBST buffer, the membrane was incubated with the peroxidase-conjugated anti-rabbit secondary antibody $(1: 4,000$; Abcam; cat. no. ab205718) for $90 \mathrm{~min}$ at room temperature. Following treatment with enhanced chemiluminescence reagent (Thermo Fisher Scientific, Inc.), the Image Lab software (version 4.1; Bio-Rad Laboratories, Inc.) was used to quantitate the band intensities. The antibodies against $\beta$-actin (cat. no. ab8227), GRP78 (cat. no. ab21685), ATF4 (cat. no. ab184909), CHOP (cat. no. ab10444), PERK (cat. no. ab65142), acH4K5 (cat. no. ab51997) and acH4K12 (cat. no. ab46983) were purchased from Abcam. The antibody against acH4 (cat. no. 39925) was purchased from Active Motif, Inc. the antibody against p-PERK (cat. no. AF4499) was purchased from Affinity Biosciences.

Reverse transcription-quantitative (RT-q)PCR. Total RNA was isolated from HepG2 cells using a TRIzol RNA 
Table I. DNA sequences of primers used for RT- and ChIP-quantitative PCR.

\begin{tabular}{lll}
\hline Gene & \multicolumn{1}{c}{ Forward (5'-3') } & Reverse $\left(5^{\prime}-3^{\prime}\right)$ \\
\hline $\begin{array}{l}\text { RT-qPCR } \\
\text { GRP78 }\end{array}$ & TTCATCTTGCCAGCCAGTTG \\
PERK & GCCTGTATTTCTAGACCTGCC & AACAACTCCAAAGCCACCAC \\
ATF4 & CTCACAGGCAAAGGAAGGAG & ACCCATGAGGTTTGAAGTGC \\
CHOP & GACCGAAATGAGCTTCCTGA & GCTCTGGGAGGTGCTTGTGA \\
$\beta$-actin & CTGCTTCTCTGGCTTGGCTG & ACTCCTGCTTGCTGATCCAC \\
ChIP-qPCR & GCACCCAGCACAATGAAGAT & \\
GRP78 & & GAGGCATTTCCGCTGGTAAC \\
ATF4 & GGGATGGAGGAAGGGAGAAC & AACACATCCACCACTGC \\
CHOP & GGTGGGTTCCATGGTCAAAT & ACTGGAGTGGTGTGGCAATG \\
\hline
\end{tabular}

RT, reverse transcription; ChIP, chromatin immunoprecipitation; F, forward; R, reverse; GRP78, 78 kDa glucose-regulated protein; ATF4, activating transcription factor 4; CHOP, C/EBP-homologous protein; PERK, PRKR-like endoplasmic reticulum kinase.

isolation kit (Thermo Fisher Scientific, Inc.) and the purity and concentration of the RNA samples were determined by a DN2000 ultramicro nucleic acid analyzer (Thermo Fisher Scientific, Inc.). The total RNA was reverse transcribed into cDNA using a First Strand cDNA Synthesis kit (Thermo Fisher Scientific, Inc), according to the manufacturer's instructions. RT-qPCR reactions were performed as follows: $25^{\circ} \mathrm{C}$ for $10 \mathrm{~min}, 48^{\circ} \mathrm{C}$ for $60 \mathrm{~min}$ and $95^{\circ} \mathrm{C}$ for $5 \mathrm{~min}$. The qPCR was performed using SYBR green mix from Thermo Fisher Scientific, Inc. The reaction conditions included an initial pre-denaturation step at $95^{\circ} \mathrm{C}$ for $30 \mathrm{sec}$, followed by 40 cycles of thermal steps consisting of $95^{\circ} \mathrm{C}$ for $5 \mathrm{sec}$ and $60^{\circ} \mathrm{C}$ for $30 \mathrm{sec} . \beta$-Actin was used as an internal control. The fold-change was calculated by the $2^{-\Delta \Delta C q}$ method (23). The primer sequences used for each gene are shown in Table I.

ChIP-qPCR. HepG2 cells $\left(1 \times 10^{6}\right)$ were seeded onto $10-\mathrm{cm}$ diameter dishes and were mock-treated with DMSO $(0 \mu \mathrm{M}$ SAHA) or treated with $6 \mu \mathrm{M}$ SAHA for $36 \mathrm{~h}$. Following treatment, the cells were subjected to a ChIP assay using a ChIP kit (Sigma-Aldrich; Merck KGaA), according to the manufacturer's instructions. The HepG2 cells were cross-linked with $1 \%$ formaldehyde and lysed with $600 \mu \mathrm{l}$ of radioimmunoprecipitation assay lysis buffer (Thermo Fisher Scientific, Inc.), and the lysates were sonicated to fragment the DNA into lengths between 200-1,000 base pairs. Immunoprecipitation was performed with the following antibodies: Anti-acH4 (1:50; Active Motif, Inc.; cat. no. 39925), rabbit IgG (1:50; Abcam; cat. no. ab205718) and anti-RNA Polymerase II (1:50; Abcam; cat. no. ab51462) at $4^{\circ} \mathrm{C}$ overnight. Protein G-sepharose beads were used for immunoprecipitation, and the immunoprecipitates were washed and eluted, according to the manufacturer's protocol. The immunoprecipitated DNA was recovered by reversing the cross-linking, and were purified and dissolved in distilled water. A corresponding sample handled without the addition of any antibody served as an input control. The ChIP DNA and input DNA were analyzed by qPCR. The abundance of the immunoprecipitated target DNA was expressed as a percentage of the input chromatin DNA. The primers used for detecting the promoter regions of the GRP78, ATF4 and CHOP genes are listed in Table I.

Statistical analysis. SPSS version 20 statistical software (IBM Corp.) was used for statistical analysis. Data were expressed as the mean \pm SD. The one-way analysis of variance method was used for the multivariate comparison, and the least significant difference method was used as a post hoc test. $\mathrm{P}<0.05$ was considered to indicate a statistically significant difference.

\section{Results}

SAHA inhibits the proliferation of Hep 2 cells in a dose-and time-dependent manner. Firstly, the effect of SAHA on the proliferation of HepG2 cells was determined by real-time cellular analysis. This involved live monitoring of cell proliferation in a label-free manner. The electrical impedance of adherent cells was detected by the electrode at the bottom of an E-plate 16, and the cell index is a dimensionless parameter that was translated from the measured electrical impedance to denote cell proliferation. After HepG 2 cells were incubated for $10 \mathrm{~h}$ to allow cell adhesion, SAHA was added at various doses in the culture medium to treat cells. This resulted in a rapid decrease in HepG2 cell proliferation at $10 \mathrm{~h}$ (Fig. 1), which was probably due to the transient effect of SAHA vehicle DMSO on cell proliferation. Compared with the mock-treated group (0 $\mu \mathrm{M}$ SAHA), treatment with SAHA at concentrations $>1 \mu \mathrm{M}$ resulted in a notable inhibitory effect on the proliferation of HepG2 cells (Fig. 1). Furthermore, SAHA at $6 \mu \mathrm{M}$ resulted in significantly $(\mathrm{P}<0.01)$ decreased normalized cell index, by $>70 \%$, at $50 \mathrm{~h}$ after treatment. A significant $(\mathrm{P}<0.01)$ suppression of proliferation was observed in HepG2 cells treated with higher doses of SAHA $(12,25$ and $50 \mu \mathrm{M})$ for longer periods of time. Thus, the antiproliferative effect of SAHA on HepG2 cells occurs in a dose- and time-dependent manner.

SAHA induces apoptosis in HepG2 cells in a dose-dependent manner. The effect of SAHA on apoptosis in HepG2 cells was investigated by annexin V-FITC and PI staining. Following 


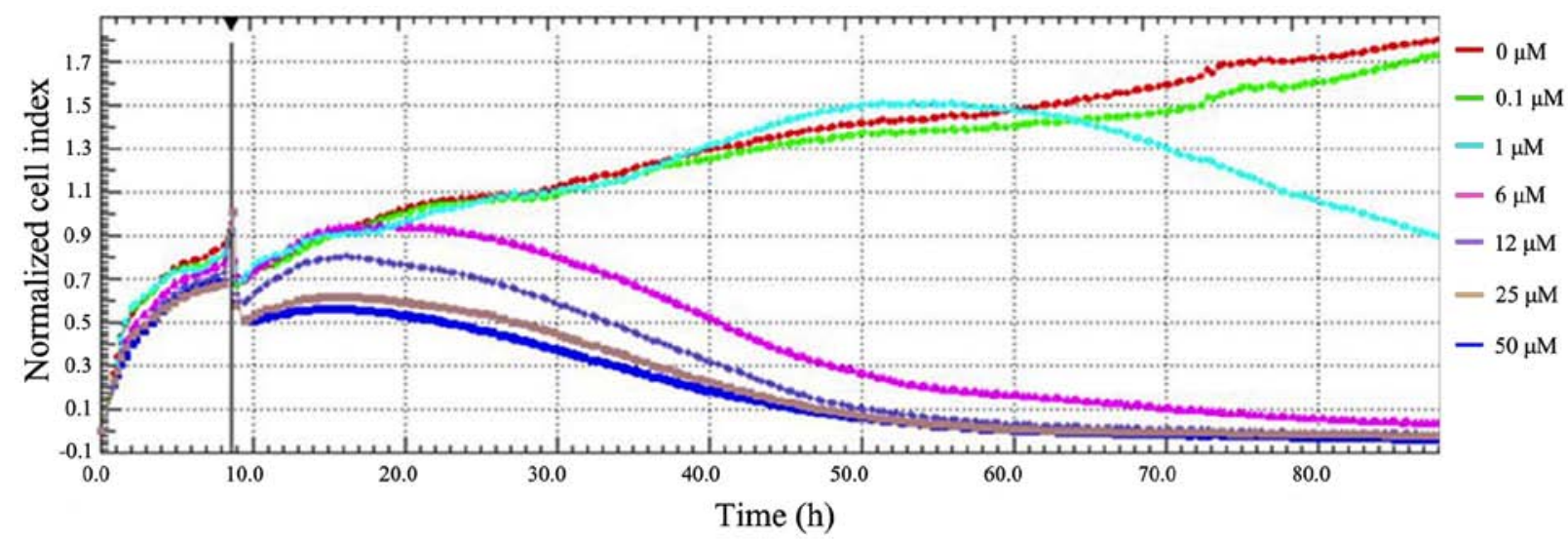

Figure 1. SAHA inhibits the proliferation of HepG2 cells in a dose- and time-dependent manner. HepG2 cells were exposed to SAHA at the indicated concentrations for $90 \mathrm{~h}$. Cell proliferation was measured by a real-time cellular analysis system. The normalized cell index is a parameter with no dimension that is translated from the electrical impedance and indicates cell proliferation. Data shown represent one of three experiments. SAHA, suberoylanilide hydroxamic acid.

treatment with SAHA for $48 \mathrm{~h}$, the apoptotic rates were significantly elevated in HepG2 cells treated with 1,6 or $12 \mu \mathrm{M}$ SAHA, compared with the untreated cells (Fig. 2). While the control group ( $0 \mu \mathrm{M}$ SAHA) had an average apoptotic rate of $\sim 1 \%, 12 \mu \mathrm{M}$ SAHA led to an increased apoptotic cell population to $\sim 10 \%$. These results indicate that SAHA could significantly promote apoptosis in HepG2 cells in a dose-dependent manner.

SAHA induces ER stress-mediated apoptotic signaling pathway in HepG2 cells. Since SAHA was revealed to be a potential inducer of ER stress, the effect of SAHA on ER stress in HepG2 cells was determined by measuring the levels of ER stress-associated molecules, including GRP78, PERK, p-PERK, ATF4 and CHOP. No significant changes were observed in the expression of GRP78 at either the mRNA (Fig. 3A) or protein level (Fig. 3B and C) in cells treated with $1 \mu \mathrm{M}$ SAHA, whereas cells treated with 6 and $12 \mu \mathrm{M}$ SAHA had significantly increased levels of GRP78 mRNA and protein compared with control cells (0 $\mu \mathrm{M}$ SAHA). No significant changes in the level of PERK mRNA were observed following treatment with any of the tested doses of SAHA (Fig. 3A). However, the expression of PERK protein was significantly decreased in the HepG2 cells treated with different concentrations of SAHA, while the level of p-PERK protein was significantly increased (Fig. 3B and C), which resulted in an increased ratio of p-PERK/PERK following SAHA treatments (Fig. 3D). Notably, following treatment with SAHA, the mRNA and protein expression levels of ATF4 and CHOP were significantly elevated in a dose-dependent manner (Fig. 3A and C). Moreover, the protein levels of ATF4 and CHOP in the HepG2 cells were significantly upregulated by over 3-fold following treatment with $12 \mu \mathrm{M}$ SAHA.

SAHA significantly upregulates the level of acH4 in HepG2 cells. The role of SAHA, as an HDACi, in the acetylation of histone $\mathrm{H} 4$ was determined by western blot analysis. The protein levels of total acH4, acH4K5 and acH4K12 were detected in HepG2 cells treated at various doses of SAHA for $48 \mathrm{~h}$ (Fig. 4). Compared with the control group (0 $\mu \mathrm{M}$ SAHA), the levels of acH4 and acH4K12 were not significantly increased in the HepG 2 cells treated with $1 \mu \mathrm{M}$ SAHA, whereas the level of acH4K5 was significantly increased; these findings were likely due to the varied sensitivities of histone deacetylases to inhibition by SAHA. In cells treated with 6 and $12 \mu \mathrm{M}$ SAHA, the levels of total acH4, acH4K5 and acH4K12 were all significantly higher than in those treated with 0 and $1 \mu \mathrm{M}$ SAHA. These results suggest that $6 \mu \mathrm{M}$ SAHA was sufficient to markedly elevate the acetylation of histone $\mathrm{H} 4$.

SAHA treatment enhances the acetylation level of histone $\mathrm{H4}$ in the promoter regions of the GRP78, ATF4 and CHOP genes in HepG2 cells. In order to confirm whether the regulation of transcription of GRP78, ATF4 and CHOP genes by SAHA is mediated by the upregulation of acH4, ChIP assays were conducted. This involved determination and quantitation of the acH4-associated promoter regions of these genes in HepG2 cells (Fig. 5). Following treatment with DMSO (0 $\mu \mathrm{M}$ SAHA) or $6 \mu \mathrm{M}$ SAHA for $36 \mathrm{~h}$, the HepG2 cells were lysed and immunoprecipitated with specific anti-acH4 antibody. qPCR results demonstrated significant increase in the acH4-associated promoter regions of the GRP78, ATF4 and CHOP genes in HepG2 cells treated with $6 \mu \mathrm{M}$ SAHA. The promoter regions of ATF4 and CHOP were increased by 2-fold and 3-fold, respectively, the promoter region of GRP78 was enriched by $\sim 4$-fold (the output to input ratio increased from $\sim 15$ to $\sim 60 \%$ ). These results confirmed that SAHA induces the expression of ER stress-associated molecules by increasing their transcription, at least partially through enhancing the acetylation of histone $\mathrm{H} 4$ in the promoter regions of these genes.

\section{Discussion}

Epigenetic therapy using an HDACi, alone or in combination with other treatments, has shown therapeutic potential in clinical trials for the treatment of several types of cancer, including non-small cell lung cancer and breast cancer $(24,25)$. As an HDACi, SAHA specifically induces tumor cells to undergo apoptosis by regulating the expression of key genes involved in apoptotic signaling 

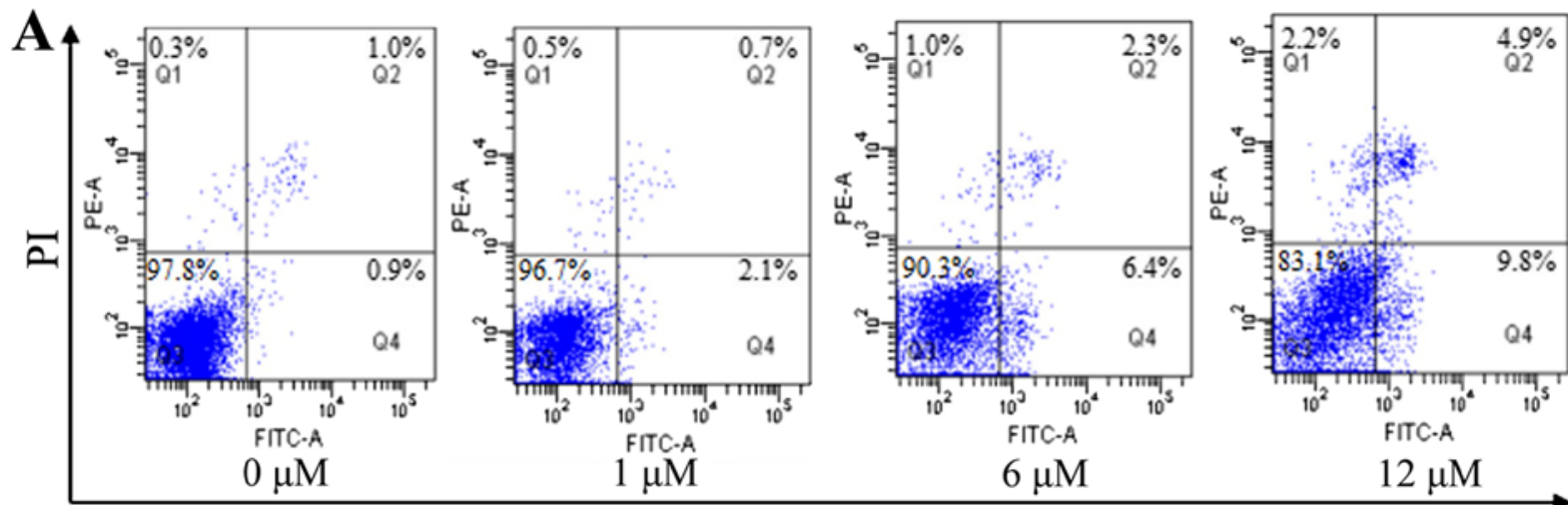

FITC-Annexin V

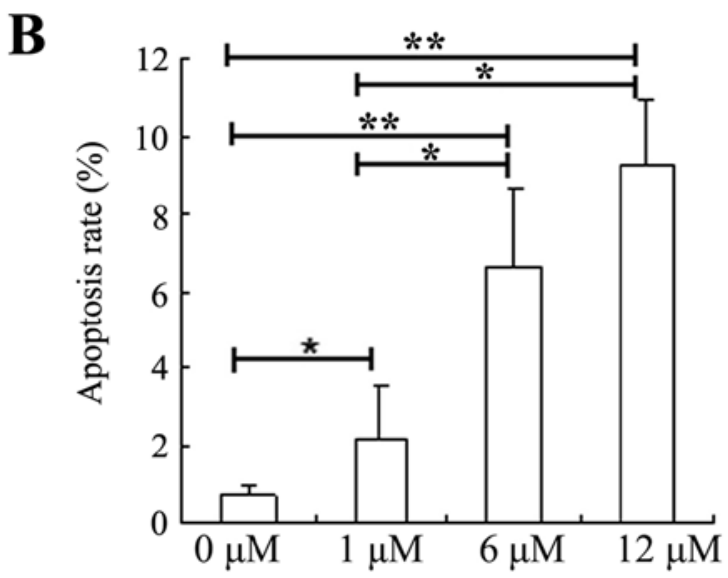

Figure 2. SAHA induces apoptosis in HepG2 cells in a dose-dependent manner. (A) Representative flow cytometry plots showing the population of apoptotic HepG2 cells treated with the specified doses of SAHA for $48 \mathrm{~h}$. Cells were stained with annexin V-FITC and PI to assess apoptosis by flow cytometry. Data shown represent one of three experiments. The lower right quadrant represents the early apoptotic cells, and the upper right quadrant represents the late apoptotic and dead cells. (B) Quantification of the apoptotic rates (percentages of Annexin V-positive cells) in HepG2 cells exposed to SAHA at the indicated doses for $48 \mathrm{~h}$. $\mathrm{N}=3$ for each group. ${ }^{*} \mathrm{P}<0.05,{ }^{* *} \mathrm{P}<0.01$. SAHA, suberoylanilide hydroxamic acid; FITC, fluorescein isothiocyanate; PI, propidium iodide; $\mathrm{Q}$, quadrant.

pathways (26). The present study revealed that SAHA induced apoptosis in HepG2 liver cancer cells by activating the ER stress-mediated apoptotic signaling pathway, at least partially through enhancing the acetylation of histone $\mathrm{H} 4$ on the promoter regions of ER-stress associated genes, including GRP78, ATF4 and CHOP. This suggests that ER stress-mediated apoptotic signaling may play a central role in HDACi-induced apoptosis of HepG 2 cells and that SAHA alone or in combination with inducers of ER stress can potentially be applied in patients with liver cancer.

SAHA-induced apoptosis has been demonstrated to be associated with activation of the intrinsic apoptotic pathways (27). It can be concluded that in tumor cells exposed to SAHA, proapoptotic genes (such as Bax and Bim) are upregulated, whereas the expression levels of antiapoptotic genes (such as Bcl-2 and Bcl-XL) are suppressed (28). SAHA has also been shown to influence the expression of death receptors (such as Fas and TNF-related apoptosis-inducing ligand receptor) and death receptor ligands in leukemia, which are responsible for the extrinsic apoptotic pathways (29). SAHA was also demonstrated to activate the ER stress-associated apoptotic signaling pathway. Kim et al (30) demonstrated increased expression levels of p-PERK, ATF4 and CHOP in papillary thyroid cancer and anaplastic thyroid cancer cells treated with SAHA, and increased apoptotic rates of these cells. However, the mechanism of SAHA on regulating the expression of the ER stress signaling pathway-related molecules remains unclear.

Certain studies have shown the inhibitory effect of SAHA on the proliferation of liver cancer cells and the induction of apoptosis $(31,32)$. Furthermore, another study demonstrated significant inhibition of proliferation of MHCC97L hepatocellular carcinoma cells in vitro by SAHA (Cai et al, unpublished data). In the present study, SAHA was demonstrated to suppress cell proliferation and induce apoptosis in a dose-dependent manner in HepG2 cells. Notably, the expression of GRP78 at both the mRNA and protein levels in HepG2 cells was significantly increased following treatment with 6 or $12 \mu \mathrm{M}$ SAHA. Since GRP78 is a protein marker of ER stress (33), its increased expression suggests that SAHA may induce ER stress in HepG2 cells. In addition, it was found that the mRNA level of PERK was unaffected by SAHA treatment, indicating that SAHA does not regulate the expression of PERK at the transcriptional level. Nevertheless, treatment with SAHA resulted in significantly decreased expression of PERK and increased expression of p-PERK in HepG2 cells at protein level. This may be because SAHA-induced HepG2 cells undergo ER stress, upon which GRP78 changes its binding preference towards unfolded/misfolded proteins accumulated in the ER, resulting in the release of PERK (34). 


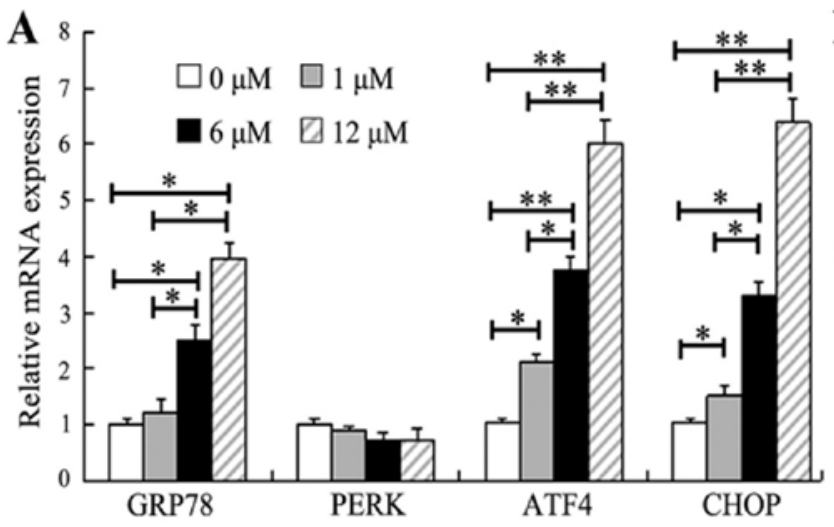

C

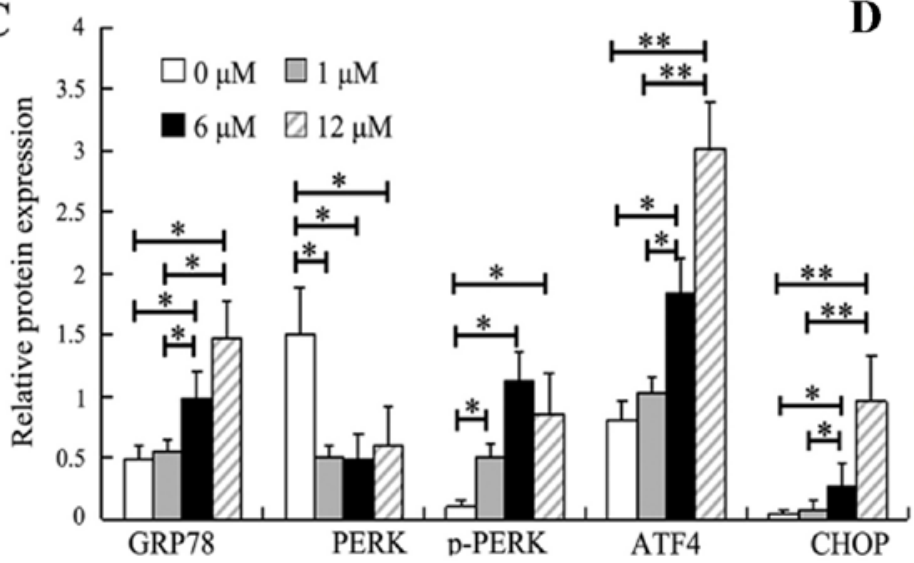

B

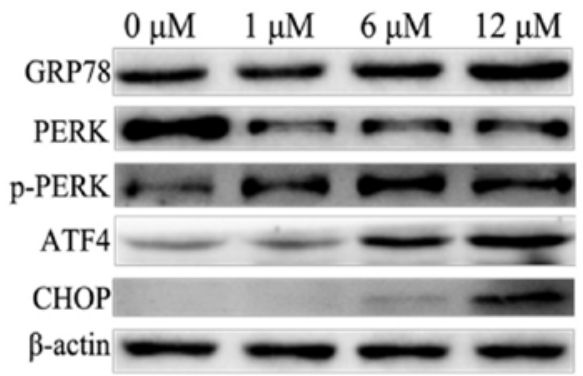

D

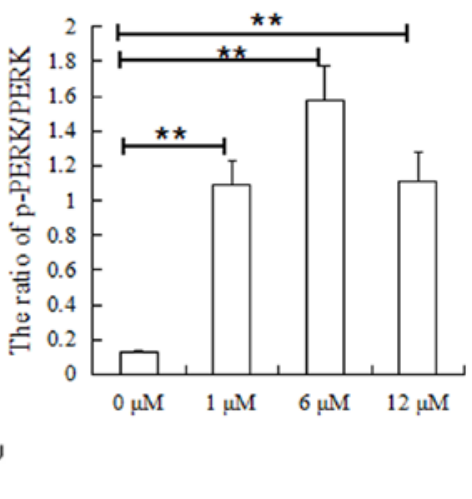

Figure 3. SAHA induces ER stress in HepG2 cells. (A) HepG2 cells were treated with SAHA at the indicated concentrations for $48 \mathrm{~h}$, and the mRNA levels of endoplasmic reticulum stress-associated genes (GRP78, PERK, ATF4 and CHOP) were analyzed by reverse transcription-quantitative PCR. (B and C) Representative images (one of three experiments) showing the (B) western blot analysis of GRP78, PERK, p-PERK, ATF4 and CHOP protein expression in HepG2 cells exposed to SAHA at the indicated concentrations and (C) their relative protein expression levels. (D) Relative ratio of p-PERK/PERK. $\mathrm{N}=3$. ${ }^{*} \mathrm{P}<0.05,{ }^{* *} \mathrm{P}<0.01$. SAHA, suberoylanilide hydroxamic acid; GRP78, $78 \mathrm{kDa}$ glucose-regulated protein; ATF4, activating transcription factor 4; CHOP, C/EBP-homologous protein; PERK, PRKR-like endoplasmic reticulum kinase; p, phosphorylated.

A
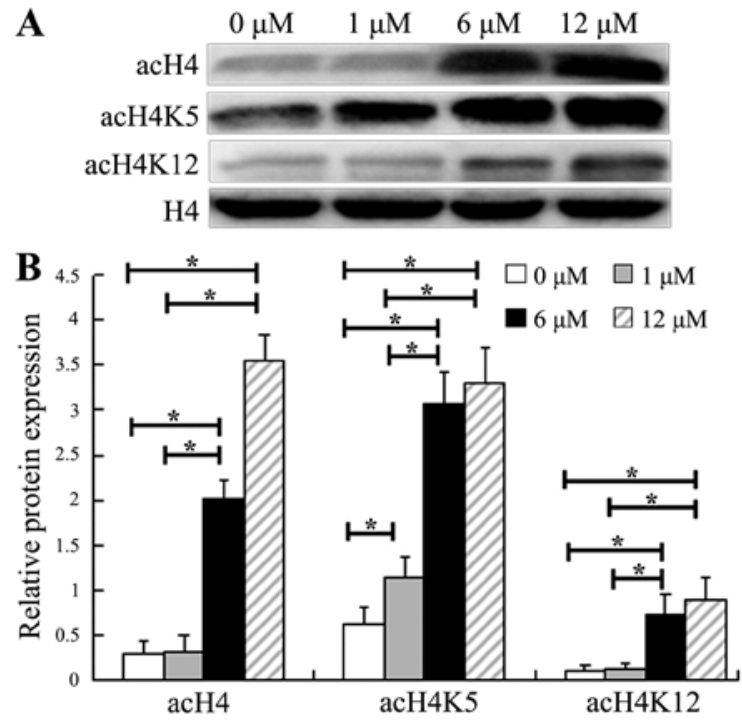

Figure 4. SAHA increases the expression of acH4, acH4K5 and acH4K12 in HepG2 cells. (A and B) Representative western blot images (one of three experiments) showing (A) the expression of acH4, acH4K5 acH4K12 and total histone $\mathrm{H} 4$ in $\mathrm{HepG} 2$ cells treated with SAHA at the indicated concentrations $(0,1,6$ and $12 \mu \mathrm{M})$ for $48 \mathrm{~h}$ and (B) their relative protein expression levels normalized to total histone $\mathrm{H} 4$. Relative protein expression indicates the ratio of the target protein $(\mathrm{acH} 4, \mathrm{acH} 4 \mathrm{~K} 5$ and acH4K12) level to the protein level of total histone $\mathrm{H} 4 . \mathrm{N}=3$. " $\mathrm{P}<0.05$. SAHA, suberoylanilide hydroxamic acid; acH4, total acetylated histone $\mathrm{H} 4$; acH4K5, acetylated histone H4-lysine 5; acH4K12, acetylated histone H4-lysine 12.

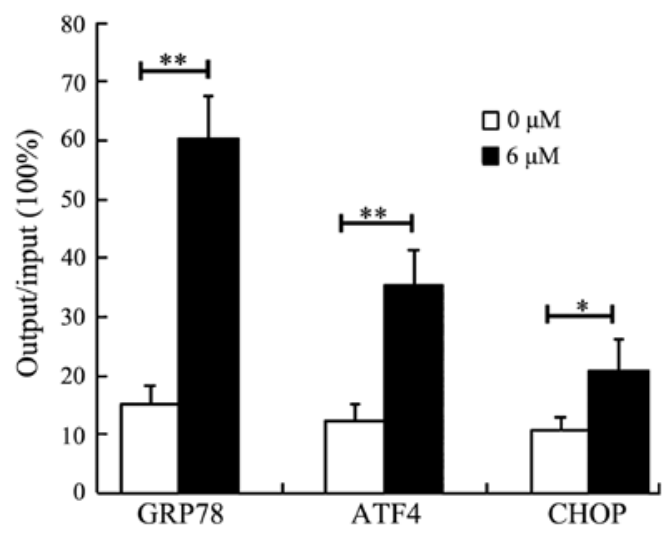

Figure 5. SAHA increases the acetylation level of histone $\mathrm{H} 4$ in the promoter regions of the GRP78, ATF4 and CHOP genes in HepG2 cells. HepG2 cells were mock-treated ( $0 \mu \mathrm{M} \mathrm{SAHA})$ or treated with $6 \mu \mathrm{M}$ SAHA for $36 \mathrm{~h}$, and the interactions with the promoter regions of these genes were measured by chromatin immunoprecipitation and qPCR assay. The data represent the output/input ratios of acetylated histone $\mathrm{H} 4$-associated DNA targets. $\mathrm{N}=3 .{ }^{*} \mathrm{P}<0.05,{ }^{* *} \mathrm{P}<0.01$. SAHA, suberoylanilide hydroxamic acid; GRP78, $78 \mathrm{kDa}$ glucose-regulated protein; ATF4, activating transcription factor 4 ; CHOP, C/EBP-homologous protein.

Following the release from GRP78, PERK may undergo self-phosphorylation and dimerization (35-37), which may lead to a decreased level of PERK protein and an increased level 
of p-PERK protein. Studies have demonstrated that p-PERK can upregulate the expression levels of ATF4 and CHOP in cells and activate the ER stress-induced apoptotic signaling pathway (38). In the present study, the increased expression of ATF4 and CHOP, at both the mRNA and protein levels, and augmented apoptosis were observed in HepG2 cells. These results suggest that induction of apoptosis in HepG2 cells by SAHA can be partially attributed to the activation of the PERK-ATF4-CHOP signaling pathway. However, it is worth noting that $12 \mu \mathrm{M}$ SAHA resulted in significantly decreased proliferation of HepG2 cells (Fig. 1), however apoptosis was only induced in $10 \%$ of HepG2 cells treated for $48 \mathrm{~h}$ (Fig. 2). This suggests that SAHA may also induce cell death in other ways to inhibit tumor cell proliferation. For example, another study demonstrated that SAHA induced autophagic death of HepG2 cells in vitro (Cai et al, unpublished data).

The present study also unveiled an unappreciated mechanism of SAHA-mediated activation of the PERK-ATF4-CHOP signaling pathway in HepG2 cells. It was inferred that this may be associated with the essential role of SAHA, as an HDACi, in the upregulation of histone acetylation. Changes in histone acetylation influence chromatin condensation, and these alterations affect gene transcription (39). In previous studies, it was found that SAHA significantly upregulated the acetylation levels of histones H3K9 and H3K27 in HepG2 cells (40). In the present study, SAHA was identified to be able to upregulate the acetylation levels of histones H4, H4K5 and H4K12, at concentrations of 6 and $12 \mu \mathrm{M}$. In addition, it was reported that the acetylation levels of histone $\mathrm{H} 3$ in the promoter region of the GRP78 gene was significantly increased, following treatment with an HDACi and thereby promoting the genetic transcription of GRP78 (41). However, it was not clear whether the increased acetylation level of $\mathrm{H} 4$ regulates the transcription of genes associated with the ER stress-mediated apoptotic signaling pathway. The ChIP-qPCR results in the present study suggest that the acetylation levels of histone $\mathrm{H} 4$ in the gene promoter regions of GRP78, ATF4 and CHOP were all significantly increased, which was associated with significantly increased mRNA levels of these genes.

In conclusion, the HDACi SAHA induces apoptosis in HepG2 cells by activating the ER stress-mediated apoptotic pathway, at least partially through upregulation of the acH4 level on the promoter regions of the ER stress-associated molecules GRP78, ATF4 and CHOP. Further studies will be conducted to investigate the effect of acetylation modification at different sites of $\mathrm{H} 4$ on the expression of molecules associated with the ER stress-mediated apoptotic pathway. In addition, a study that aims to demonstrate whether SAHA has the same inhibitory effect in a mouse xenograft model in vivo will also be conducted. Overall, the present study underscores the critical roles of ER stress in mediating apoptosis in HepG2 cells and also suggests the potential application of SAHA and other inducers of ER stress for the treatment of patients with liver cancer.

\section{Acknowledgements}

The authors would like to thank Mrs Xue Shen (Department of Central Laboratory, The Affiliated Hospital of Guizhou Medical University) for technical help and advice on the flow cytometry experiments, the Basic Medical Science Research Center of Guizhou Medical University for providing the xCELLigence Real-Time Cell Analysis instrument, and Dr Tengxiang Chen (Department of Physiology, College of Basic Medical Sciences, Guizhou Medical University) for critical reading of the manuscript and suggestions.

\section{Funding}

The present study was supported by the National Natural Science Foundation of China (grant no. 81560105), the Foundation of the Department of Science and Technology [grant no. LH (2014) 7074] and the Natural Science Foundation of the Department of Education [grant no. KY (2014) 269].

\section{Availability of data and materials}

The datasets used and/or analyzed during the present study are available from the corresponding author on reasonable request.

\section{Authors' contributions}

LY, TT, LZ and LT performed the majority of the experiments. $\mathrm{BH}, \mathrm{SC}, \mathrm{ZM}$ and TY provided analytical tools and performed the statistical analysis. RX and QY designed the study. RX and $\mathrm{BH}$ provided financial support for this work and wrote the manuscript. All authors read and approved the final manuscript.

\section{Ethics approval and consent to participate}

Not applicable.

\section{Patient consent for publication}

Not applicable.

\section{Competing interests}

The authors declare that they have no competing interests.

\section{References}

1. Buurman R, Sandbothe M, Schlegelberger B and Skawran B: HDAC inhibition activates the apoptosome via Apaf1 upregulation in hepatocellular carcinoma. Eur J Med Res 21: 26, 2016.

2. Chen QW, Zhu XY, Li YY and Meng ZQ: Epigenetic regulation and cancer (review). Oncol Rep 31: 523-532, 2014.

3. Muhammad JS, Khan MR and Ghias K: DNA methylation as an epigenetic regulator of gallbladder cancer: An overview. Int J Surg 53: 178-183, 2018.

4. Shanmugam MK, Arfuso F, Arumugam S, Chinnathambi A, Jinsong B, Warrier S, Wang LZ, Kumar AP, Ahn KS, Sethi G and Lakshmanan M: Role of novel histone modifications in cancer. Oncotarget 9: 11414-11426, 2017.

5. Khan FS, Ali I, Afridi UK, Ishtiaq M and Mehmood R: Epigenetic mechanisms regulating the development of hepatocellular carcinoma and their promise for therapeutics. Hepatol Int 11: 45-53, 2017.

6. Wang Y, Yan L, Zhang Z, Prado E, Fu L, Xu X and Du L: Epigenetic regulation and its therapeutic potential in pulmonary hypertension. Front Pharmacol 9: 241, 2018.

7. Peng L and Zhong X: Epigenetic regulation of drug metabolism and transport. Acta Pharm Sin B 5: 106-112, 2015.

8. Liu KY, Wang LT and Hsu SH: Modification of epigenetic histone acetylation in hepatocellular Carcinoma. Cancers (Basel) 10: pii: E8, 2018. 
9. Reddy D, Khade B, Pandya R and Gupta S: A novel method for isolation of histones from serum and its implications in therapeutics and prognosis of solid tumours. Clin Epigenetics 9: 30, 2017.

10. Vahid F, Zand H, Nosrat-Mirshekarlou E, Najafi R and Hekmatdoost A: The role dietary of bioactive compounds on the regulation of histone acetylases and deacetylases: A review. Gene 562: 8-15, 2015.

11. Schneider A, Chatterjee S, Bousiges O, Selvi BR, Swaminathan A, Cassel R, Blanc F, Kundu TK and Boutillier AL: Acetyltransferases (HATs) as targets for neurological therapeutics. Neurotherapeutics 10: 568-588, 2013.

12. Zhou Y, Peng J and Jiang S: Role of histone acetyltransferases and histone deacetylases in adipocyte differentiation and adipogenesis. Eur J Cell Biol 93: 170-177, 2014.

13. Chrun ES, Modolo F and Daniel FI: Histone modifications: A review about the presence of this epigenetic phenomenon in carcinogenesis. Pathol Res Pract 213: 1329-1339, 2017.

14. Kanno K, Kanno S, Nitta H, Uesugi N, Sugai T, Masuda T, Wakabayashi G and Maesawa C: Overexpression of histone deacetylase 6 contributes to accelerated migration and invasion activity of hepatocellular carcinoma cells. Oncol Rep 28 : 867-873, 2012.

15. Liu W, Xiao J, Lan J, et al: The effects of histone acetylation on the migration and invasion of hepatocellular carcinoma cells. J Guizhou Med Univ 42: 1365-1369, 2017 (In Chinese).

16. Mrakovcic M, Kleinheinz J and Fröhlich LF: Histone deacetylase inhibitor-induced autophagy in tumor cells: Implications for p53. Int J Mol Sci 18: pii: E1883, 2017.

17. Wu Z, Jing S, Li Y, Gao Y, Yu S, Li Z, Zhao Y, Piao J, Ma S and Chen X: The effects of SAHA on radiosensitivity in pancreatic cancer cells by inducing apoptosis and targeting RAD51. Biomed Pharmacother 89: 705-710, 2017.

18. Lu H, Yang XF, Tian XQ, Tang SL, Li LQ, Zhao S and Zheng HC: The in vitro and vivo anti-tumor effects and molecular mechanisms of suberoylanilide hydroxamic acid (SAHA) and MG132 on the aggressive phenotypes of gastric cancer cells. Oncotarget 7 : $56508-56525,2016$.

19. Xue K, Gu JJ, Zhang Q, Mavis C, Hernandez-Ilizaliturri FJ, Czuczman MS and Guo Y: Vorinostat, a histone deacetylase (HDAC) inhibitor, promotes cell cycle arrest and re-sensitizes rituximab-and chemo-resistant lymphoma cells to chemotherapy agents. J Cancer Res Clin Oncol 142: 379-387, 2016.

20. Hanke NT, Garland LL and Baker AF: Carfilzomib combined with suberanilohydroxamic acid (SAHA) synergistically promotes endoplasmic reticulum stress in non-small cell lung cancer cell lines. J Cancer Res Clin Oncol 142: 549-560, 2016.

21. Teng Z, Kuang X, Wang J and Zhang X: Real-time cell analysis-a new method for dynamic, quantitative measurement of infectious viruses and antiserum neutralizing activity. J Virol Methods 193 364-370, 2013.

22. Zandi K: A real-time cell analyzing assay for identification of novel antiviral compounds against chikungunya virus. Methods Mol Biol 1426: 255-262, 2016.

23. Livak KJ and Schmittgen TD: Analysis of relative gene expression data using real-time quantitative PCR and the 2(-Delta Delta C(T)) method. Methods 25: 402-408, 2001.

24. Lakshmaiah KC, Jacob LA, Aparna S, Lokanatha D and Saldanha SC: Epigenetic therapy of cancer with histone deacetylase inhibitors. J Cancer Res Ther 10: 469-478, 2014.

25. Ahuja N, Sharma AR and Baylin SB: Epigenetic therapeutics: A new weapon in the war against cancer. Annu Rev Med 67: 73-89, 2016.

26. Hurwitz JL, Stasik I, Kerr EM, Holohan C, Redmond KM, McLaughlin KM, Busacca S, Barbone D, Broaddus VC, Gray SG, et al: Vorinostat/SAHA-induced apoptosis in malignant mesothelioma is FLIP/caspase 8-dependent and HR23B-independent. Eur J Cancer 48: 1096-1107, 2012.
27. Yamamoto S, Tanaka K, Sakimura R, Okada T, Nakamura T, Li Y, Takasaki M, Nakabeppu Y and Iwamoto Y: Suberoylanilide hydroxamic acid (SAHA) induces apoptosis or autophagy-associated cell death in chondrosarcoma cell lines. Anticancer Res 28: 1585-1591, 2008

28. Hrabeta J, Stiborova M, Adam V, Kizek R and Eckschlager T: Histone deacetylase inhibitors in cancer therapy. A review. Biomed Pap Med Fac Univ Palacky Olomouc Czech Repub 158: 161-169, 2014.

29. Arhoma A, Chantry AD, Haywood-Small SL and Cross NA: SAHA-induced TRAIL-sensitisation of multiple myeloma cells is enhanced in 3D cell culture. Exp Cell Res 360: 226-235, 2017.

30. Kim SM, Park KC, Jeon JY, Kim BW, Kim HK, Chang HJ, Choi SH, Park CS and Chang HS: Potential anti-cancer effect of N-hydroxy-7-(2-naphthylthio) heptanomide (HNHA), a novel histone deacetylase inhibitor, for the treatment of thyroid cancer. BMC Cancer 15: 1003, 2015.

31. Li YL, Zhang NY, Hu X, Chen JL, Rao MJ, Wu LW, Li QY, Zhang B, Yan W and Zhang C: Evodiamine induces apoptosis and promotes hepatocellular carcinoma cell death induced by vorinostat via downregulating HIF-1 $\alpha$ under hypoxia. Biochem Biophys Res Commun 498: 481-486, 2018.

32. Kunnimalaiyaan S, Sokolowski K, Gamblin TC and Kunnimalaiyaan M: Suberoylanilide hydroxamic Acid, a histone deacetylase inhibitor, alters multiple signaling pathways in hepatocellular carcinoma cell lines. Am J Surg 213: 645-651, 2017.

33. Matsuo K, Gray MJ, Yang DY, Srivastava SA, Tripathi PB, Sonoda LA, Yoo EJ, Dubeau L, Lee AS and Lin YG: The endoplasmic reticulum stress marker, glucose-regulated protein-78 (GRP78) in visceral adipocytes predicts endometrial cancer progression and patient survival. Gynecol Oncol 128: 552-559, 2013.

34. Shi-Chen Ou D, Lee SB, Chu CS, Chang LH, Chung BC and Juan LJ: Transcriptional activation of endoplasmic reticulum chaperone GRP78 by HCMV IE1-72 protein. Cell Res 21: 642-653, 2011.

35. Wang M, Law ME, Castellano RK and Law BK: The unfolded protein response as a target for anticancer therapeutics. Crit Rev Oncol Hematol 127: 66-79, 2018.

36. Yoo YS, Han HG and Jeon YJ: Unfolded protein response of the endoplasmic reticulum in tumor progression and immunogenicity. Oxid Med Cell Longev 2017: 2969271, 2017.

37. Nakka VP, Prakash-Babu P and Vemuganti R: Crosstalk between endoplasmic reticulum stress, oxidative stress, and autophagy: Potential therapeutic targets for acute CNS injuries. Mol Neurobiol 53: 532-544, 2016.

38. Chen Y, Gui D, Chen J, He D, Luo Y and Wang N: Down-regulation of PERK-ATF4-CHOP pathway by Astragaloside IV is associated with the inhibition of endoplasmic reticulum stress-induced podocyte apoptosis in diabetic rats. Cell Physiol Biochem 33: 1975-1987, 2014.

39. Ketchum CC, Larsen CD, McNeil A, Meyer-Ficca ML and Meyer RG: Early histone H4 acetylation during chromatin remodeling in equine spermatogenesis. Biol Reprod 98: 115-129, 2018.

40. Lei YU, Han B, Tian T, Zheng L, Yang T, Liu X, Tang L, Luo X, Yang Q and Xie JR: Suberoylanilide hydroxamic acid induces apoptosis of HepG2 cells by endoplasmic reticulum stress apoptotic pathway. Chin J Pathophysiol 33: 2151-2156, 2017.

41. Chen M, Liu Q, Chen L, Zhang L and Gu E: Remifentanil postconditioning ameliorates histone $\mathrm{H} 3$ acetylation modification in $\mathrm{H} 9 \mathrm{c} 2$ cardiomyoblasts after hypoxia/reoxygenation via attenuating endoplasmic reticulum stress. Apoptosis 22: 662-671, 2017.

This work is licensed under a Creative Commons Attribution-NonCommercial-NoDerivatives 4.0 International (CC BY-NC-ND 4.0) License. 